\title{
Florística e fitossociologia do componente arbóreo-arbustivo de mata ciliar em estágio inicial de sucessão na microbacia do rio Xaxim (Oeste do Paraná, Brasil): subsídios para a restauração ecológica
}

\author{
Floristic and phytosociology of the tree-shrub component of \\ riparian forest in early successional stage in the Xaxim river \\ microbasin (Western Paraná, Brazil): subsidies for ecological \\ restoration
}

\author{
Lisboa, Thais de F. B. ${ }^{*}$ (1); Roque Cielo-Filho2 ${ }^{(1)}$; Carla D. Câmara1 (D) \\ 1 Universidade Tecnológica Federal do Paraná (UTFPR), Campus Medianeira, Av. Brasil, 4232, Parque \\ Independência, 85884-000, Medianeira, Paraná, Brasil. \\ 2 Secretaria de Infraestrutura e Meio Ambiente do Estado de São Paulo, Instituto Florestal, Rua \\ Pernambuco, s/n, 18701-180, Avaré, São Paulo, Brasil. \\ * Autor correspondente: thais.lisboa7@hotmail.com
}

\section{RESUMO}

A megabiodiversidade existente no Brasil pode ser observada em uma variedade de biomas, como a Mata Atlântica, que é considerada um dos biomas mais biodiversos do mundo. Mas essa biodiversidade vem sendo ameaçada por atividades antrópicas que resultam em fragmentação de habitats e degradação ambiental. Técnicas preconizadas pela ciência da restauração ecológica têm sido aplicadas como modo de reverter a degradação ambiental e promover a restauração da vegetação. Informações qualitativas e quantitativas sobre a vegetação de áreas em processo de sucessão secundária constituem subsídios importantes para a aplicação de técnicas de restauração ativa, baseadas no plantio de mudas. Este trabalho apresenta um estudo florístico e fitossociológico do componente arbóreo-arbustivo de vegetação ciliar com 12 anos de idade que regenerou espontaneamente em área de antiga pastagem (restauração passiva). Foram compiladas informações quanto à abundância das espécies, síndrome de dispersão (zoocoria, anemocoria e autocoria), categoria sucessional (pioneiras e

\footnotetext{
> Ref. bibliográfica: Lisboa, T. de F. B., Cielo-Filho, R., Câmara, C. D. (2021). Florística e fitossociologia do componente arbóreo-arbustivo de mata ciliar em estágio inicial de sucessão na microbacia do rio Xaxim (Oeste do Paraná, Brasil): subsídios para a restauração ecológica. Lilloa 58 (1): 15-34. doi: https://doi. org/10.30550/j.lil/2021.58.1/2021.04.07

> Recibido: 5 de marzo 2020 - Aceptado: 7 de abril 2021- Publicado en línea: 21 de abril 2021.

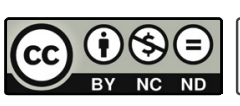

${ }_{\text {OPEN }}{ }_{\text {ACCESS }}$ 
não-pioneiras) e ameaça de extinção. Foram amostrados 856 indivíduos, e identificadas 60 espécies nativas e quatro espécies exóticas distribuídas em 31 famílias. As famílias mais abundantes foram Myrtaceae, Euphorbiaceae e Fabaceae, com 431 indivíduos, 175 indivíduos e 60 indivíduos, respectivamente. As famílias mais ricas foram Myrtaceae e Fabaceae, com 11 e sete espécies, respectivamente. As espécies mais abundantes e frequentes foram Myrcia selloi, Gymnanthes klotzschiana, Myrcia subcordata, Sebastiania brasiliensis, Myrcia glomerata e Eugenia involucrata. A síndrome de dispersão zoocórica foi a mais comum, com 38 espécies e 547 indivíduos. A categoria sucessional não-pioneira predominou com 33 espécies e 533 indivíduos. Não foram encontradas espécies ameaçadas de extinção na área. As informações disponibilizadas neste estudo poderão subsidiar projetos de restauração ativa de matas ciliares na região.

Palavras-chave - Biodiversidade; conservação; flora; successão; Mata Atlântica.

\section{ABSTRACT}

The existing megabiodiversity in Brazil is represented a variety of biomes, such as the Atlantic Forest, which is considered one of the most biodiverse biomes in the world. However, this biodiversity has been threatened by human activities, resulting in habitat fragmentation and environmental degradation. Techniques recommended by ecological restoration science have been applied to reverse environmental degradation and promote the restoration of vegetation. Qualitative and quantitative information on the vegetation of areas in the process of secondary succession constitutes important subsidies for the application of active restoration techniques based on the planting of seedlings. This work presents a floristic and phytosociological study of the tree-shrub component of 12-year-old riparian vegetation that spontaneously regenerated in an area of abandoned pasture (passive restoration). Information regarding species abundance, dispersal syndrome (zoochory, anemochory and autochory), successional category (pioneer and non-pioneer) and extinction threaten was compiled. A total of 856 individuals were sampled, where 60 native species and four exotic ones were identified, distributed in 31 families. The most abundant families were Myrtaceae, Euphorbiaceae and Fabaceae, with 431, 175 and 60 individuals, respectively. The richest families were Myrtaceae and Fabaceae with 11 and seven species, respectively. The most abundant and frequent species were Myrcia selloi, Gymnanthes klotzschiana, Myrcia subcordata, Sebastiania brasiliensis, Myrcia glomerata and Eugenia involucrata. The zoochoric dispersal syndrome was the most common with 38 species and 547 individuals. The non-pioneer successional category predominated with 33 species and 533 individuals. No endangered species were found in the area. The information available in this study may support projects for the active restoration of riparian forests in the region.

Keywords - Atlantic Forest; biodiversity; conservation; flora; succession. 


\section{INTRODUÇÃO}

A megabiodiversidade existente no Brasil pode ser observada em uma variedade de biomas, como a Mata Atlântica, que por sua elevada diversidade florística e faunística, bem como pelo alto grau de endemismo, é considerada um dos biomas mais biodiversos do mundo (Myers, Mittermeier, Mittermeier, Fonseca, Kent, 2000; Forzza et al. 2012).

Contudo, essa biodiversidade vem sendo ameaçada por atividades antrópicas como a rápida expansão da agricultura, urbanização, exploração insustentável dos recursos naturais, dentre outras atividades; resultando em fragmentação de habitats e degradação ambiental (Di Bitetti, Placci, Dietz, 2003).

Um exemplo desse processo de fragmentação e degradação vem ocorrendo ao longo dos anos no estado do Paraná, onde a cobertura florestal nativa foi reduzida a $18,51 \%$ da área ocupada originalmente (Accioly, 2013). A Floresta Estacional Semidecidual, principal formação florestal das regiões Oeste, Noroeste e Norte do Estado, foi a mais devastada, restando apenas $6,07 \%$ da sua cobertura original, constituídos, majoritariamente, por pequenos fragmentos florestais (Accioly, 2013).

A supressão e fragmentação de habitats têm se revelado como graves ameaças à biodiversidade, colocando em risco a sobrevivência de muitas espécies (Tossulino et al., 2007; Muchailh, Roderjan, Campos, Machado, Curcio, 2010). As matas ciliares também têm sofrido com os impactos causados pela fragmentação florestal, pois as pressões antrópicas são intensificadas em locais próximos a ambientes aquáticos (Tossulino et al., 2007).

A vegetação ciliar no Paraná vem sendo transformada em um mosaico de fragmentos florestais (Borghi, Martins, Del Quiqui, Nanni, 2004), e isto leva a grandes preocupações ambientais, visto que essa tipologia florestal desempenha importantes funções ecológicas como a proteção dos recursos hídricos; auxílio na regulação do ciclo hidrológico; manutenção da qualidade da água (Tundisi e Tundisi, 2010); e o fornecimento de abrigo, alimento e rotas de deslocamento para a fauna (Mello, Petri, Leite, Toppa, 2014).

No Brasil, em razão das importantes funções desempenhadas pelas matas ciliares, elas são legalmente consideradas como "Áreas de Preservação Permanente (APP's)" e são delimitadas faixas adjacentes aos corpos d'água para sua proteção, além de ser obrigatória a restauração da vegetação ciliar que tenha sofrido supressão (Brasil, 2012; Chaves, Durigan, Brancalion, Aronson, 2015). Como modo de reverter a degradação ambiental e promover a restauração dessa vegetação, aplicam-se técnicas preconizadas pela ciência da restauração ecológica, com o objetivo de dar início e/ou acelerar a recuperação de ecossistemas degradados (Society for Ecological Restoration [SER], 2004).

A restauração passiva é caracterizada por promover o retorno espontâneo da vegetação de uma área degradada por meio da sucessão secundária, sem necessidade de auxílio humano deliberado (Aronson, Durigan, Brancalion, 2011). Áreas restauradas passivamente, por processos naturais, especialmente aquelas em estágios sucessionais iniciais, são particularmente relevantes para a obtenção de informações ecológicas com potencial de aplicação na restauração da vegetação ciliar. Deste modo, 
informações florísticas e fitossociológicas de áreas em processo de restauração passiva (baseada na regeneração natural) configuram contribuições importantes para a aplicação de técnicas de restauração ativa (baseadas no plantio de mudas) em outras áreas degradadas geograficamente próximas, mas que não apresentam resiliência suficiente para desencadear seus processos de restauração naturalmente.

A restauração florestal atrelada à implantação de corredores ecológicos é uma estratégia importante para reverter os processos de fragmentação florestal, pois promove o aumento da conectividade entre fragmentos, facilitando o trânsito de espécies e o restabelecimento de fluxos biológicos (Muchailh et al., 2010). A região Oeste do estado do Paraná é considerada estratégica para a implantação de corredores ecológicos em escala local, de modo a viabilizar a implantação em uma escala bem mais ampla, do corredor de biodiversidade do Rio Paraná, conectando os remanescentes de Mata Atlântica localizados na Argentina, Paraguai e Brasil (Di Bitetti et al., 2003). A restauração da vegetação ciliar pode contribuir significativamente para a implantação desses corredores ecológicos em escala local, devido ao efeito benéfico exercido por essa vegetação na conectividade ecológica da paisagem (Seganfredo, Cunha, Magalhães, Cielo-Filho, Câmara, 2019). Somado a isso, a conservação da vegetação ciliar é de fundamental importância na gestão de bacias hidrográficas, uma vez que sua remoção pode intensificar a degradação de mananciais gerando aumento de custos de tratamento da água para abastecimento, dentre outros múltiplos prejuízos ambientais e econômicos (Tundisi e Tundisi, 2010).

Estudos em remanescentes de mata ciliar específicos de cada tipologia vegetacional são fundamentais na reversão dos processos de degradação (Borghi et al., 2004). Apesar de remanescentes florestais resultantes do processo de fragmentação apresentarem amostras pouco representativas da vegetação primária, o conhecimento acerca de sua diversidade é fundamental para aumentar a efetividade das ações de conservação e restauração (Machado et al., 2008).

Diante disso, este trabalho apresenta um estudo florístico e fitossociológico do componente arbóreo-arbustivo de mata ciliar resultante do processo de restauração passiva e em estágio inicial de sucessão, em área localizada na microbacia do rio Xaxim, região Oeste do Paraná, Brasil, incluindo informações ecológicas quanto à composição e abundância das espécies, síndrome de dispersão, categoria sucessional e ameaça de extinção.

\section{MATERIAIS E MÉTODOS}

\section{Área de estudo}

A área de estudo situa-se a uma altitude média de $489 \mathrm{~m}$ acima do nível do mar, nas coordenadas $25^{\circ} 07^{\prime} 42.46^{\prime \prime S}$ e 5356'53.09”O (Fig. 1). Encontra-se no limite entre os municípios de Matelândia e Céu Azul, estado do Paraná, a cerca de $10 \mathrm{~km}$ de distância do maior remanescente de vegetação nativa da região, o Parque Nacional do Iguaçu (PNI). O tipo de solo predominante na área é o Nitossolo (Bhering et al., 2007) e o clima na região é caracterizado como subtropical úmido, Cfa, na classificação de Köppen (Alvares, Stape, Sentelhas, Gonçalves, Sparovek, 2013). Apresenta 
precipitação média anual variando de 1600 a $1800 \mathrm{~mm}$, e temperatura média anual de $21,6{ }^{\circ} \mathrm{C}$, com média mínima anual menor que $17^{\circ} \mathrm{C}$ e média máxima anual maior que $28^{\circ} \mathrm{C}$ (Nitsche, Caramori, Ricce, Pinto, 2019).

A formação florestal predominante na região é a Floresta Estacional Semidecidual (Hornes e Rodrigues, 2018), pertencente à Ecorregião Florestas do Alto Paraná, a qual possui um dos maiores blocos de floresta remanescente de Mata Atlântica (Di Bitetti et al., 2003). Esta tipologia vegetal é influenciada pelo clima subtropical, que lhe confere a característica de semidecidualidade com queda parcial das folhas no período do inverno (Instituto Brasileiro de Geografia e Estatística [IBGE], 2012). A vegetação ciliar da área integra a microbacia do rio Xaxim e pode ser classificada como Floresta Estacional Semidecidual Montana, visto que a área se situa na faixa altimétrica de 400 a $1.000 \mathrm{~m}$, acima de $24^{\circ}$ de latitude Sul (IBGE, 2012); e ribeirinha com influência fluvial sazonal devido a sua localização em ambiente ripário com inundações eventuais (Rodrigues, 2000).

A área selecionada, apesar de ter sofrido durante anos com impactos provenientes de pastoreio por gado, apresentou resiliência suficiente para dar início ao processo de sucessão secundária, após isolamento da área (Lisboa, Cielo-Filho, Câmara, 2021), apresentando-se atualmente em estágio sucessional inicial. A estratégia de restauração passiva foi aplicada na área em 2005, e compreendeu a instalação de cercas para isolamento de faixas de aproximadamente $30 \mathrm{~m}$ de largura às margens do corpo hídrico, como forma de possibilitar a regeneração natural da vegetação ciliar.

\section{Amostragem e coleta de dados}

Os dados foram coletados no período de novembro de 2017 a julho de 2018. A amostragem florística e fitossociológica foi realizada por meio da alocação de 10 parcelas de $5 \mathrm{~m} \times 5 \mathrm{~m}$ cada, as quais foram posicionadas sistematicamente a cada $45 \mathrm{~m}$ entre si, paralelamente e a uma distância mínima de $5 \mathrm{~m}$ da margem do corpo hídrico, resultando em uma área total amostrada de $250 \mathrm{~m}^{2}$. Dentro de cada parcela foi realizada a identificação e contagem dos indivíduos de espécies arbóreas e/ou arbustivas que apresentaram altura igual ou superior a $0,5 \mathrm{~m}$.

\section{Identificação e caracterização de espécies amostradas}

Foi realizada a coleta de material botânico e a identificação das espécies foi feita até o menor nível taxonômico possível por meio de comparações com exsicatas depositadas no herbário Figueira (FIG), da Universidade Tecnológica Federal do Paraná; e consulta a literatura especializada (e.g. Ramos, Durigan, Franco, Siqueira, Rodrigues, 2015). A coleção do herbário FIG é constituída essencialmente por materiais coletados em um estudo florístico mais amplo realizado pelos autores em fragmentos de floresta nativa situados nos arredores do PNI (Cielo-Filho et al., 2017a). Assim, a identificação botânica neste trabalho foi beneficiada pelo conhecimento prévio da flora regional obtido pelos autores. As espécies foram ordenadas em famílias de acor- 

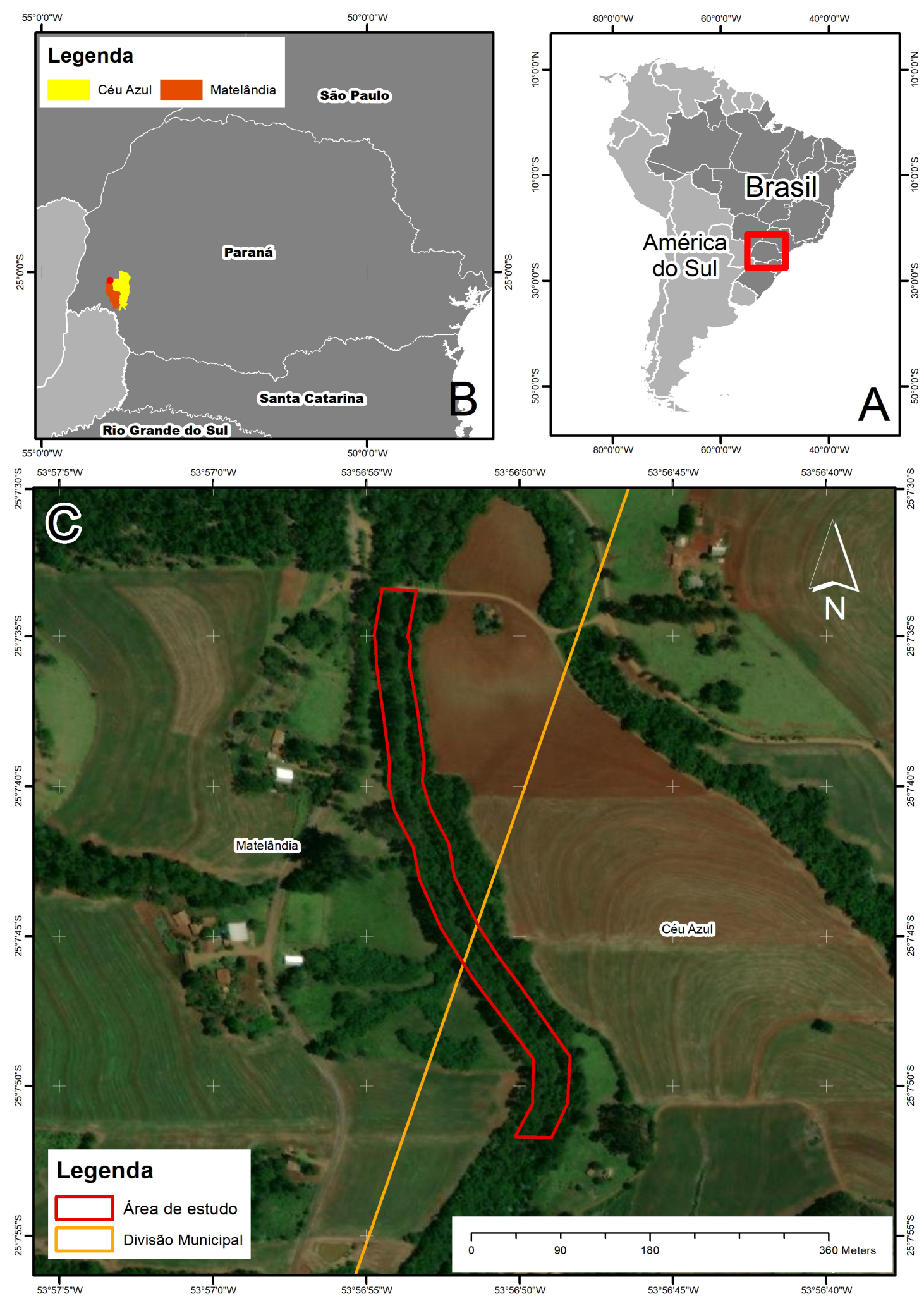

Fig. 1. Mapa de localização da área de estudo. A) Mapa da América do Sul com destaque para o Brasil. B) Mapa do estado do Paraná com destaque para a localização dos municípios limítrofes Céu Azul e Matelândia. C) Delimitação da área de estudo.

Fig. 1. Location map of the study area. A) Map of South America with emphasis on Brazil. B) Map of the state of Paraná with emphasis on the location of the municipalities bordering Céu Azul and Matelândia. C) Delimitation of the study area. 
do com o sistema APG IV (Angiosperm Phylogeny Group [APG], 2016). As sinonímias botânicas, grafias e abreviações foram verificadas na Lista de Espécies da Flora do Brasil (Flora do Brasil 2020, 2021).

A partir do banco de dados fornecido por Barbosa et al. (2017), foram obtidas informações para cada espécie quanto à síndrome de dispersão [zoocoria, anemocoria e autocoria - sensu Van der Pijl (1982)] e categoria sucessional [pioneiras e não-pioneiras - sensu Whitmore (1989)]. Informações relativas ao hábito (arbóreas e arbustivas) e a origem (nativas e exóticas) das espécies identificadas foram consultadas na base de dados da Lista de Espécies da Flora do Brasil (Flora do Brasil 2020, 2021). A informação sobre o hábito da espécie Syagrus romanzoffiana, divergiu da informação contida naquela base de dados, pois optou-se por adotar o hábito definido para essa espécie em Barbosa et al. (2017). Os hábitos dos indivíduos que não puderam ser identificados ao nível de espécie foram definidos conforme observações em campo. Também foram verificadas as espécies ameaçadas de extinção tomando como base a Lista Nacional Oficial de Espécies da Flora Ameaçadas de Extinção (Brasil, 2014); bem como as espécies invasoras conforme a Lista de Espécies Exóticas Invasoras do Estado do Paraná (Paraná, 2015) e a Base de dados de espécies exóticas invasoras do Brasil (Instituto Hórus de Desenvolvimento e Conservação Ambiental, 2021).

Conforme metodologias usualmente adotadas em levantamentos florísticos, as espécies exóticas e as que não puderam ser identificadas ao nível de espécie, não foram caracterizadas quanto ao hábito, síndrome de dispersão ou categoria sucessional.

Com vistas a conhecer a distribuição e abundância das espécies, foram calculados os valores absolutos e relativos para densidade e frequência das espécies amostradas na área de estudo (Moro e Martins, 2011; Freitas e Magalhães, 2012). Por meio do somatório da densidade e frequência relativas foi possível obter um valor que expressa conjuntamente a abundância e a distribuição de espécies na área, aqui denominado como índice de regularidade horizontal (IRH), com base em Freitas e Magalhães (2012). Também foi calculado o índice de diversidade de Shannon (H') (Ludwig e Reynolds, 1988).

\section{RESULTADOS}

Foram contabilizados 856 indivíduos de espécies arbóreas e/ou arbustivas, as quais foram identificadas em sua maioria ao nível de espécie, algumas ao nível de gênero (Piper sp. - 14 indivíduos e Solanum sp. - um indivíduo) ou família (Myrtaceae - um indivíduo) e uma espécie indeterminada (três indivíduos); totalizando 64 táxons distribuídos em 31 famílias (Tabela 1). Destas espécies, quatro são consideradas exóticas (Tabela 2).

Nenhuma das espécies amostradas se encontra em ameaça de extinção, segundo a Lista Nacional Oficial de Espécies da Flora Ameaçadas de Extinção (Brasil, 2014). 
Tabela 1. Relação de espécies arbóreas e arbustivas com características ecológicas. N IND (número de indivíduos); arb = arbusto, arv = árvore; SD (síndrome de dispersão): ANE = anemocórica, AUT = autocórica, $Z \mathrm{ZOO}=$ zoocórica; CS (categoria sucessional): $\mathrm{P}=$ pioneira, NP = não-pioneira.

Table 1. List of tree and shrub species with ecological characteristics. No IND (number of individuals); $\mathrm{arb}=$ shrub, arv = tree; SD (dispersal syndrome): ANE = anemochoric, $\mathrm{AUT}=$ autochoric, $\mathrm{ZOO}=$ zoochoric; CS (successional category): $\mathrm{P}=$ pioneer, NP = non-pioneer.

\begin{tabular}{|c|c|c|c|c|}
\hline FAMÍLIA / Espécie & $\mathbf{N}^{\circ}$ IND & Hábito & SD & CS \\
\hline $\begin{array}{l}\text { ACANTHACEAE } \\
\text { Aphelandra schottiana (Ness) Profice }\end{array}$ & 35 & arb & AUT & NP \\
\hline $\begin{array}{l}\text { ANNONACEAE } \\
\text { Annona emarginata (Schltdl.) H.Rainer }\end{array}$ & 1 & arv & ZOO & $\mathrm{P}$ \\
\hline $\begin{array}{l}\text { ARECACEAE } \\
\text { Syagrus romanzoffiana (Cham.) Glassman }\end{array}$ & 2 & arv & zoo & NP \\
\hline $\begin{array}{l}\text { ASPARAGACEAE } \\
\text { Cordyline spectabilis Kunth \& Bouché }\end{array}$ & 2 & arv & zoo & $\mathrm{P}$ \\
\hline $\begin{array}{l}\text { ASTERACEAE } \\
\text { Dasyphyllum brasiliense (Spreng.) Cabrera }\end{array}$ & 1 & arb, arv & ANE & NP \\
\hline $\begin{array}{l}\text { BIGNONIACEAE } \\
\text { Jacaranda micrantha Cham. }\end{array}$ & 2 & arv & ANE & $\mathrm{P}$ \\
\hline $\begin{array}{l}\text { BORAGINACEAE } \\
\text { Cordia trichotoma (Vell.) Arráb. ex Steud. }\end{array}$ & 1 & arv & ANE & NP \\
\hline $\begin{array}{l}\text { ERYTHROXYLACEAE } \\
\text { Erythroxylum myrsinites Mart. }\end{array}$ & 14 & arb, arv & zoo & NP \\
\hline $\begin{array}{l}\text { EUPHORBIACEAE } \\
\text { Bernardia pulchella (Baill.) Müll.Arg. }\end{array}$ & 1 & arb & AUT & $P$ \\
\hline Gymnanthes klotzschiana Müll.Arg. & 122 & arb, arv & AUT & NP \\
\hline Sebastiania brasiliensis Spreng. & 52 & arb, arv & AUT & $\mathrm{P}$ \\
\hline $\begin{array}{l}\text { FABACEAE } \\
\text { Calliandra foliolosa Benth. }\end{array}$ & 36 & arb, arv & AUT & NP \\
\hline Dalbergia frutescens (Vell.) Britton & 3 & arb & ANE & NP \\
\hline Machaerium paraguariense Hassl. & 1 & arv & ANE & NP \\
\hline $\begin{array}{l}\text { Muellera campestris (Mart. ex Benth.) M.J. Silva \& } \\
\text { A.M.G. Azevedo }\end{array}$ & 5 & arv & AUT & NP \\
\hline Parapiptadenia rigida (Benth.) Brenan & 12 & arv & AUT & NP \\
\hline Peltophorum dubium (Spreng.) Taub. & 1 & arv & AUT & $\mathrm{P}$ \\
\hline Senegalia polyphylla (DC.) Britton \& Rose & 2 & arb, arv & AUT & $\mathrm{P}$ \\
\hline $\begin{array}{l}\text { LAMINACEAE } \\
\text { Aegiphila brachiata Vell. }\end{array}$ & 1 & arb, arv & ZOO & $\mathrm{P}$ \\
\hline $\begin{array}{l}\text { LAURACEAE } \\
\text { Ocotea pulchella (Nees \& Mart.) Mez }\end{array}$ & 7 & arv & zoo & NP \\
\hline $\begin{array}{l}\text { LOGANIACEAE } \\
\text { Strychnos brasiliensis (Spreng.) Mart. }\end{array}$ & 6 & arb & zoo & NP \\
\hline $\begin{array}{l}\text { MALVACEAE } \\
\text { Guazuma ulmifolia Lam. }\end{array}$ & 1 & arv & zoo & $P$ \\
\hline Heliocarpus popayanensis Kunth. & 1 & arv & ANE & $P$ \\
\hline Luehea divaricata Mart. & 3 & arv & ANE & $P$ \\
\hline Pavonia sepium A.St.-Hil. & 2 & arb & AUT & NP \\
\hline $\begin{array}{l}\text { MELASTOMATACEAE } \\
\text { Miconia cinerascens Miq. }\end{array}$ & 4 & arb, arv & zoo & $\mathrm{P}$ \\
\hline $\begin{array}{l}\text { MELIACEAE } \\
\text { Trichilia elegans A.Juss. }\end{array}$ & 1 & arb, arv & zoo & NP \\
\hline
\end{tabular}


Tabela 1 (cont.).

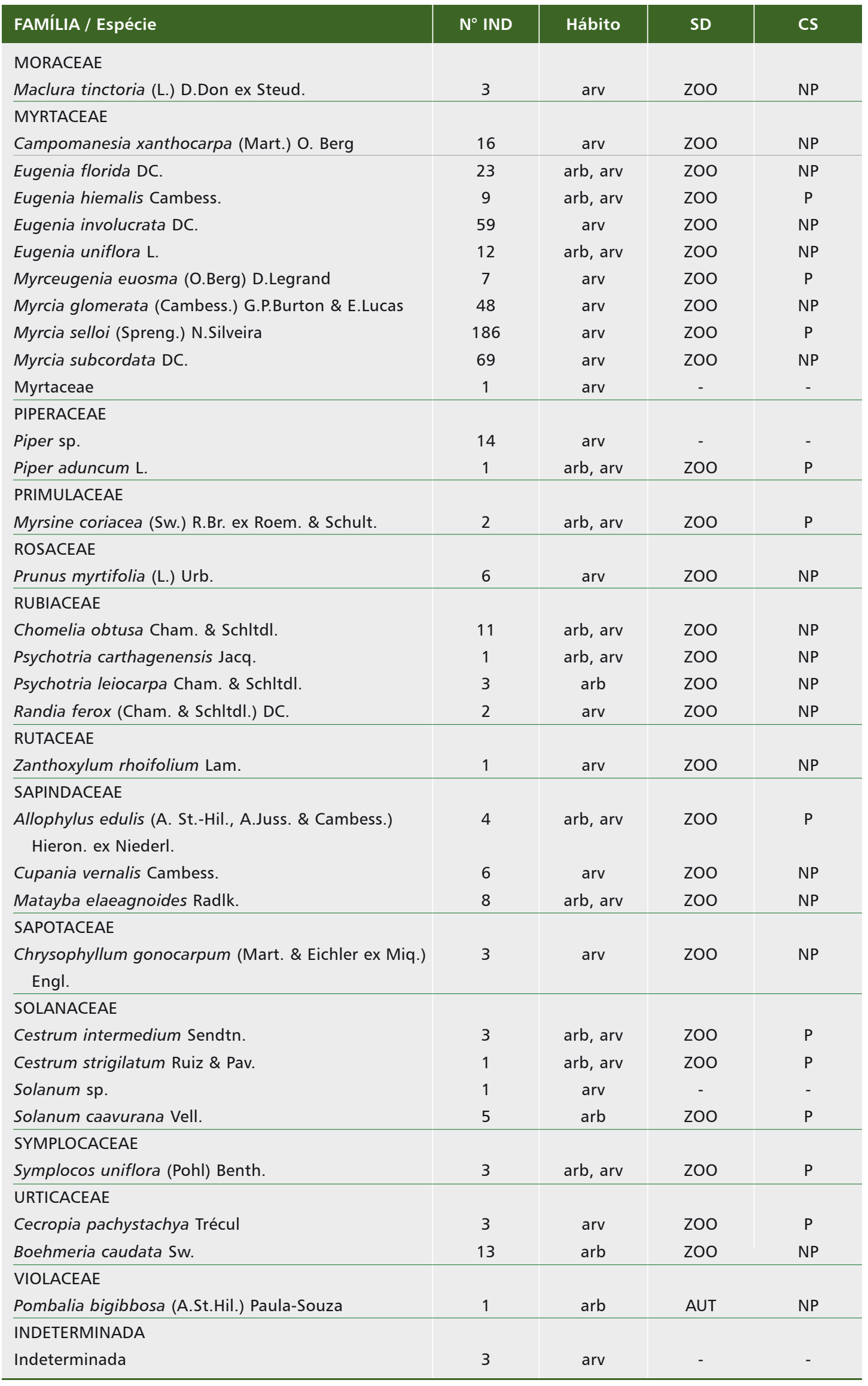


Tabela 2. Relação de espécies exóticas encontradas nas parcelas.

Table 2. List of exotic species found in the plots.

As espécies exóticas amostradas na área de estudo foram Citrus $\times$ limon, Eriobotrya japonica, Hovenia dulcis e Psidium guajava. Estas quatro espécies estão compreendidas em quatro famílias, das quais Rhamnaceae está representada exclusivamente por Hovenia dulcis (Tabela 2). Essas espécies são consideradas invasoras em níveis estadual (Paraná, 2015) e nacional (Instituto Hórus de Desenvolvimento e Conservação Ambiental, 2021).

O hábito arbóreo foi representado por 30 espécies, o hábito arbustivo por nove espécies e 21 espécies podem apresentar tanto o hábito arbóreo como arbustivo (Tabela 1).

Em relação às síndromes de dispersão, as espécies zoocóricas foram maioria (38 espécies e 547 indivíduos), seguidas pelas espécies autocóricas (11 espécies e 269 indivíduos) e anemocóricas (sete espécies e 12 indivíduos). No que se refere à categoria sucessional, houve predominância de espécies não-pioneiras (33 espécies e 533 indivíduos) em relação às espécies pioneiras (23 espécies e 295 indivíduos) (Tabela 1).

As espécies mais abundantes e frequentes na área de estudo, consideradas como aquelas com maiores valores estimados de IRH, foram Myrcia selloi $(25,41)$, Gymnanthes klotzschiana $(18,66)$, Myrcia subcordata (11,74), Sebastiania brasiliensis $(10,49)$, Myrcia glomerata $(10,02)$ e Eugenia involucrata $(9,83)$. Dos 64 táxons encontrados, $19(29,69 \%)$ foram representados por somente um indivíduo e $44(68,75 \%)$ por seis indivíduos ou menos (Tabela 3).

A família Myrtaceae foi a mais abundante, com 50,35\% dos indivíduos amostrados, seguida de Euphorbiaceae (20,44\%), e Fabaceae (7,01\%). As famílias Myrtaceae e Fabaceae também se apresentaram como as mais ricas, com 17,19\% (11 espécies) e 10,94\% (sete espécies) das espécies amostradas, respectivamente.

O Índice de Diversidade de Shannon (H') foi estimado em 2,99 nats.ind. ${ }^{-1}$. 
Tabela 3. Valores estimados para os parâmetros fitossociológicos das espécies amostradas na área de estudo, em ordem decrescente de valores de IRH. DA (densidade absoluta), DR (densidade relativa), FA (frequência absoluta), FR (frequência relativa) e IRH (índice de regularidade horizontal).

Table 3. Estimated values for the phytosociological parameters of the sampled species in the study area, in decreasing order of IRH values. DA (absolute density), DR (relative density), FA (absolute frequency), FR (relative frequency) and IRH (horizontal regularity index).

\begin{tabular}{|c|c|c|c|c|c|}
\hline FAMÍLIA / Espécie & $\begin{array}{c}\text { DA } \\
\text { (ind.ha-1) }\end{array}$ & DR (\%) & FA (\%) & FR (\%) & IRH \\
\hline Myrcia selloi (Spreng.) N.Silveira & 7440 & 21,73 & 50 & 3,68 & 25,41 \\
\hline Gymnanthes klotzschiana Müll.Arg. & 4880 & 14,25 & 60 & 4,41 & 18,66 \\
\hline Myrcia subcordata DC. & 2760 & 8,06 & 50 & 3,68 & 11,74 \\
\hline Sebastiania brasiliensis Spreng. & 2080 & 6,07 & 60 & 4,41 & 10,49 \\
\hline $\begin{array}{l}\text { Myrcia glomerata (Cambess.) G.P.Burton \& } \\
\text { E.Lucas }\end{array}$ & 1920 & 5,61 & 60 & 4,41 & 10,02 \\
\hline Eugenia involucrata DC. & 2360 & 6,89 & 40 & 2,94 & 9,83 \\
\hline Calliandra foliolosa Benth. & 1440 & 4,21 & 50 & 3,68 & 7,88 \\
\hline Aphelandra schottiana (Ness) Profice & 1400 & 4,09 & 50 & 3,68 & 7,77 \\
\hline Miconia cinerascens Miq. & 320 & 0,93 & 60 & 4,41 & 5,35 \\
\hline Campomanesia xanthocarpa (Mart.) O. Berg & 640 & 1,87 & 40 & 2,94 & 4,81 \\
\hline Eugenia florida DC. & 920 & 2,69 & 20 & 1,47 & 4,16 \\
\hline Erythroxylum myrsinites Mart. & 560 & 1,64 & 30 & 2,21 & 3,84 \\
\hline Piper sp. & 560 & 1,64 & 30 & 2,21 & 3,84 \\
\hline Eugenia uniflora L. & 480 & 1,40 & 30 & 2,21 & 3,61 \\
\hline Parapiptadenia rigida (Benth.) Brenan & 480 & 1,40 & 30 & 2,21 & 3,61 \\
\hline Chomelia obtusa Cham. \& Schltdl. & 440 & 1,29 & 30 & 2,21 & 3,49 \\
\hline Ocotea pulchella (Nees \& Mart.) Mez & 280 & 0,82 & 30 & 2,21 & 3,02 \\
\hline $\begin{array}{l}\text { Allophylus edulis (A. St.-Hil., A.Juss. \& } \\
\text { Cambess.) Hieron. ex Niederl. }\end{array}$ & 160 & 0,47 & 30 & 2,21 & 2,67 \\
\hline Citrus $\times$ limon (L.) Osbeck & 160 & 0,47 & 30 & 2,21 & 2,67 \\
\hline Indeterminada & 120 & 0,35 & 30 & 2,21 & 2,56 \\
\hline Eugenia hiemalis Cambess & 360 & 1,05 & 20 & 1,47 & 2,52 \\
\hline Myrceugenia euosma (O.Berg) D.Legrand & 280 & 0,82 & 20 & 1,47 & 2,29 \\
\hline Boehmeria caudata Sw. & 520 & 1,52 & 10 & 0,74 & 2,25 \\
\hline Prunus myrtifolia (L.) Urb. & 240 & 0,70 & 20 & 1,47 & 2,17 \\
\hline Strychnos brasiliensis (Spreng.) Mart. & 240 & 0,70 & 20 & 1,47 & 2,17 \\
\hline $\begin{array}{l}\text { Muellera campestris (Mart. ex Benth.) M.J. } \\
\text { Silva \& A.M.G. Azevedo }\end{array}$ & 160 & 0,47 & 20 & 1,47 & 1,94 \\
\hline Cestrum intermedium Sendtn. & 120 & 0,35 & 20 & 1,47 & 1,82 \\
\hline Hovenia dulcis Thunb. & 120 & 0,35 & 20 & 1,47 & 1,82 \\
\hline Machaerium paraguariense Hassl. & 120 & 0,35 & 20 & 1,47 & 1,82 \\
\hline Jacaranda micrantha Cham. & 80 & 0,23 & 20 & 1,47 & 1,70 \\
\hline Myrsine coriacea (Sw.) R.Br. ex Roem. \& Schult. & 80 & 0,23 & 20 & 1,47 & 1,70 \\
\hline Senegalia polyphylla (DC.) Britton \& Rose & 80 & 0,23 & 20 & 1,47 & 1,70 \\
\hline Cupania vernalis Cambess. & 240 & 0,70 & 10 & 0,74 & 1,44 \\
\hline Luehea divaricata Mart. & 200 & 0,58 & 10 & 0,74 & 1,32 \\
\hline Solanum caavurana Vell. & 200 & 0,58 & 10 & 0,74 & 1,32 \\
\hline Cecropia pachystachya Trécul & 120 & 0,35 & 10 & 0,74 & 1,09 \\
\hline $\begin{array}{l}\text { Chrysophyllum gonocarpum (Mart. \& Eichler } \\
\text { ex Miq.) Engl. }\end{array}$ & 120 & 0,35 & 10 & 0,74 & 1,09 \\
\hline Dalbergia frutescens (Vell.) Britton & 120 & 0,35 & 10 & 0,74 & 1,09 \\
\hline Matayba elaeagnoides Radlk. & 120 & 0,35 & 10 & 0,74 & 1,09 \\
\hline
\end{tabular}


Tabela 3 (cont.).

\begin{tabular}{|c|c|c|c|c|c|}
\hline FAMÍLIA / Espécie & $\begin{array}{c}\text { DA } \\
\text { (ind.ha-1) }\end{array}$ & DR (\%) & FA (\%) & FR (\%) & IRH \\
\hline Psychotria leiocarpa Cham. \& Schltdl. & 120 & 0,35 & 10 & 0,74 & 1,09 \\
\hline Symplocos uniflora (Pohl) Benth. & 120 & 0,35 & 10 & 0,74 & 1,09 \\
\hline Cordyline spectabilis Kunth \& Bouché & 80 & 0,23 & 10 & 0,74 & 0,97 \\
\hline Pavonia sepium A.St.-Hil. & 80 & 0,23 & 10 & 0,74 & 0,97 \\
\hline Randia ferox (Cham. \& Schltdl.) DC. & 80 & 0,23 & 10 & 0,74 & 0,97 \\
\hline Syagrus romanzoffiana (Cham.) Glassman & 80 & 0,23 & 10 & 0,74 & 0,97 \\
\hline Aegiphila brachiata Vell. & 40 & 0,12 & 10 & 0,74 & 0,85 \\
\hline Annona emarginata (Schltdl.) H.Rainer & 40 & 0,12 & 10 & 0,74 & 0,85 \\
\hline Bernardia pulchella (Baill.) Müll.Arg. & 40 & 0,12 & 10 & 0,74 & 0,85 \\
\hline Cestrum strigilatum Ruiz \& Pav. & 40 & 0,12 & 10 & 0,74 & 0,85 \\
\hline Cordia trichotoma (Vell.) Arráb. ex Steud. & 40 & 0,12 & 10 & 0,74 & 0,85 \\
\hline Dasyphyllum brasiliense (Spreng.) Cabrera & 40 & 0,12 & 10 & 0,74 & 0,85 \\
\hline Eriobotrya japonica (Thunb.) Lindl. & 40 & 0,12 & 10 & 0,74 & 0,85 \\
\hline Guazuma ulmifolia Lam. & 40 & 0,12 & 10 & 0,74 & 0,85 \\
\hline Heliocarpus popayanensis Kunth. & 40 & 0,12 & 10 & 0,74 & 0,85 \\
\hline Maclura tinctoria (L.) D.Don ex Steud. & 40 & 0,12 & 10 & 0,74 & 0,85 \\
\hline Myrtaceae & 40 & 0,12 & 10 & 0,74 & 0,85 \\
\hline Peltophorum dubium (Spreng.) Taub. & 40 & 0,12 & 10 & 0,74 & 0,85 \\
\hline Piper aduncum L. & 40 & 0,12 & 10 & 0,74 & 0,85 \\
\hline Pombalia bigibbosa (A.St.Hil.) Paula-Souza & 40 & 0,12 & 10 & 0,74 & 0,85 \\
\hline Psidium guajava L. & 40 & 0,12 & 10 & 0,74 & 0,85 \\
\hline Psychotria carthagenensis Jacq. & 40 & 0,12 & 10 & 0,74 & 0,85 \\
\hline Solanum sp. & 40 & 0,12 & 10 & 0,74 & 0,85 \\
\hline Trichilia elegans A.Juss. & 40 & 0,12 & 10 & 0,74 & 0,85 \\
\hline Zanthoxylum rhoifolium Lam. & 40 & 0,12 & 10 & 0,74 & 0,85 \\
\hline Totais & 34240 & 100 & 1360 & 100 & 200 \\
\hline
\end{tabular}

\section{DISCUSSÃO}

Todas as espécies exóticas e invasoras encontradas neste estudo também foram relatadas por Cielo-Filho et al. (2017a) em fragmentos florestais nos arredores do PNI. Na Argentina, a espécie Eriobotrya japonica também é considerada invasora havendo registros de sua ocorrência em ambientes degradados e de sua expansão para ambientes naturais, como na província de Misiones (Delucchi e Keller, 2010). Segundo Stampella, Delucchi, Pochettino (2013), Citrus x taitensis (mesmo que Citrus x limon) vem invadindo diferentes ambientes na província de Misiones, incluindo bosques de vegetação secundária, assim como constatado no presente estudo. De forma semelhante, Lazzarin et al. (2015) relataram a invasão de florestas secundárias por Hovenia dulcis na região do alto Uruguai, estado de Santa Catarina, Brasil. O potencial invasivo das espécies exóticas Hovenia dulcis e Psidium guajava é evidenciado pelo predomínio destas espécies em uma área reflorestada com plantio de espécies nativas e exóticas, em que Gris e Temponi (2017) as relataram como as espécies mais abundantes em Área de Preservação Permanente do Lago de Itaipu. Além disso, Hovenia dulcis faz parte do grupo de espécies exóticas invasoras que possuem 
qualquer forma de cultivo, transporte, propagação ou aquisição, proibida no estado do Paraná (Paraná, 2015).

Apesar da reduzida abundância de espécies exóticas (quatro espécies e nove indivíduos), é preciso observar o potencial invasivo destas espécies (Moro et al., 2012), as quais podem comprometer o processo de restauração na área. Florestas em estágios sucessionais iniciais, como a da área deste estudo, podem ser mais susceptíveis à invasão de espécies exóticas (Lazzarin et al., 2015) e, portanto, ações de controle devem ser tomadas. Assim, sugere-se que as espécies exóticas sejam suprimidas do local, de modo a prevenir que se acentue o processo de invasão e possibilitar a continuidade dos processos sucessionais da vegetação ciliar.

Alguns fatores como diferenças entre esforços amostrais, épocas de coleta e estado de conservação da vegetação influenciam os resultados de levantamentos acerca das diferentes formas de vida, e dificultam comparações precisas entre áreas (CieloFilho et al., 2009). Ainda assim, tal como no presente estudo, espécies que apresentam exclusivamente o hábito arbóreo foram predominantes em diversos levantamentos abrangendo diferentes formas de vida na Floresta Estacional Semidecidual nos estados de São Paulo e Paraná (Mikish e Silva, 2001; Yamamoto, Kinoshita, Martins, 2005; Kinoshita et al., 2006; Cielo-Filho et al., 2017a; Cielo-Filho et al., 2017b). Considerando que a predominância do hábito arbóreo é uma característica de florestas mais desenvolvidas (Mikish e Silva, 2001), os resultados obtidos sugerem o avanço de processos sucessionais na vegetação da área de estudo.

A predominância de espécies zoocóricas e não-pioneiras também foi observada em áreas em processo de restauração passiva na Floresta Estacional Semidecidual. Assim, Melo e Durigan (2007) verificaram 53,1\% de espécies zoocóricas em uma área com idade de 23 anos, e no trabalho de Viapiana, Cielo-Filho, Câmara (2019) foi observado $57 \%$ de espécies zoocóricas e 74\% de espécies não-pioneiras, em uma área com idade de 25 anos. Áreas preservadas compostas por vegetação primária próximas ao local de estudo também apresentaram a predominância de espécies não-pioneiras e zoocóricas, como relatado por Viapiana et al. (2019) em estudo realizado em uma área de referência inserida no PNI que constatou a presença de 59\% de espécies zoocóricas e 79\% de espécies não-pioneiras no local. Apesar de distar aproximadamente $10 \mathrm{~km}$ da área de estudo, sugere-se que o PNI, considerado a maior fonte de propágulos da região, possa influenciar a composição florística e riqueza de espécies da área.

A predominância de espécies zoocóricas é uma característica fundamental em áreas em restauração, pois como elas servem de fonte de alimento para a fauna, tal predominância pode acentuar a contribuição das espécies zoocóricas para o aumento da riqueza específica e complexidade estrutural (Mikish e Silva, 2001), além de indicar que há ocorrência de fluxos biológicos e o avanço do processo sucessional na área (Scariot e Reis, 2010).

Segundo Maciel, Watzlawick, Schoeninger, Yamaji (2003) as espécies pioneiras germinam em ambientes que possuem dossel aberto, recebendo radiação solar ao menos parte do dia, já as espécies não-pioneiras tendem a germinar em ambientes sombreados. Assim, florestas mais maduras tendem a fornecer um ambiente mais favorável às espécies de estágios sucessionais mais avançados. A proporção de espécies não-pioneiras registrada neste estudo (58,93\%) demonstra que a área está 
progredindo em sua trajetória sucessional, visto que florestas atlânticas podem levar cerca de 157 anos para atingir 90\% de espécies não-pioneiras (Liebsch, Marques, Goldenberg, 2008).

Myrcia selloi, espécie que apresentou o maior valor estimado de IRH neste estudo, também aparece entre as espécies com maior índice de valor de importância amostradas por Silva, Fonseca, Soares-Silva, Muller, Bianchini (1995) em floresta ciliar na bacia hidrográfica do rio Tibagi no estado do Paraná. A segunda espécie com maior valor IRH foi Gymnanthes klotzschiana, a qual é encontrada com ampla distribuição em ambientes alagáveis e ciliares em Florestas Estacionais Semideciduais nos estados do Paraná e São Paulo (Bianchini, Popolo, Dias, Pimenta, 2003; Aquino e Barbosa, 2009). A espécie Sebastiania brasiliensis que também está entre as espécies de maior IRH deste estudo, foi apontada como espécie indicadora de Floresta Estacional Semidecidual ribeirinha em estudo realizado por Souza, Machado, Galvão, Filho (2017) no PNI.

Os valores elevados de IRH das espécies Myrcia selloi, Gymnanthes klotzschiana, Myrcia subcordata, Sebastiania brasiliensis, Myrcia glomerata e Eugenia involucrata, podem ser explicados pela eventual capacidade dessas espécies se desenvolverem bem em solos hidromórficos, com lençol freático pouco profundo, ou em áreas submetidas a inundações periódicas, como na área de estudo; sendo consideradas espécies importantes para a conservação e restauração de matas ciliares na região (Kieras, Accioly, Machado, 2018; Cielo-Filho et al., 2017b). Sugere-se que essas espécies sejam priorizadas em projetos de restauração ativa de matas ciliares na região da área de estudo, inclusive utilizando maior número de indivíduos dessas espécies em plantios de restauração. Por outro lado, as demais espécies nativas encontradas com menores valores de IRH, também são importantes nesses projetos, ajudando a atingir níveis elevados de diversidade na área a ser restaurada.

A família Myrtaceae foi a mais rica neste estudo, e também aparece entre as famílias mais representativas em diversos levantamentos florísticos desenvolvidos no estado do Paraná em áreas de abrangência da Floresta Estacional Semidecidual (Mikish e Silva, 2001; Estevan, Vieira, Gorenstein, 2016; Cielo-Filho et al., 2017a) e em florestas da bacia do Rio Tibagi, compostas por Florestas Ombrófilas Mistas e Florestas Estacionais Semideciduais (Silva et al., 1995; Dias, Vieira, Paiva, 2002). Além da abundância relativamente elevada de indivíduos, também é notável a elevada frequência da maioria das espécies da família Myrtaceae. As espécies dessa família se destacam em diversos trabalhos de restauração de áreas degradadas, devido à produção de frutos que potencializam a dispersão de sementes por animais (Meira Junior, Pereira, Machado, Mota, Otoni, 2015). Myrtaceae é considerada uma importante família para restauração ecológica, devido à sua contribuição no enriquecimento florestal ao longo da sucessão secundária (Liebsch et al., 2008).

As famílias Euphorbiaceae e Fabaceae apresentam, em geral, elevada riqueza de espécies em estudos desenvolvidos em Florestas Estacionais Semideciduais (Dias $e t$ al., 2002; Kinoshita et al., 2006; Aquino e Barbosa, 2009). A família Fabaceae também figura como a família mais rica em vários levantamentos florísticos na Argentina e no Brasil (Borghi et al., 2004; Cielo-Filho et al., 2017a; Estevan et al., 2016). Em vista 
disso, tais famílias possuem significativa importância na restauração de ambientes ripários (Borghi et al., 2004).

$\mathrm{O}$ valor do índice de diversidade de Shannon (H') encontrado neste estudo (2,99 nats.ind. $\left.^{-1}\right)$ foi mais próximo do encontrado por Viapiana et al. (2019) em uma área de floresta primária inserida no PNI $(2,97$ nats.ind.-1), do que em uma área em restauração passiva (2,22 nats.ind. $\left.{ }^{-1}\right)$. $\mathrm{O}$ valor de $\mathrm{H}^{\prime}$ observado neste trabalho também foi maior do que os encontrados em ambientes florestais em estágios iniciais de sucessão, como 2,89 nats.ind..$^{-1}$ em estágio sucessional inicial em Floresta Ombrófila Mista (Oliva et al., 2018); 2,65 nats.ind..$^{-1}$ em fragmento reflorestado em estágio inicial em Floresta Estacional Semidecidual (Gris e Temponi, 2017); 2,16 nats.ind..$^{-1} \mathrm{e}$ 2,66 nats.ind..$^{-1}$ em estratos de regeneração em estágio inicial em região de transição entre Floresta Ombrófila Densa e Floresta Ombrófila Mista (Souto e Boeger, 2011). Salienta-se, contudo, que tais comparações não são precisas, visto que as referidas áreas possuem diferentes históricos de uso, idades e critérios de amostragem.

Ainda assim, é possível verificar que a área de estudo apresenta uma diversidade de espécies relativamente elevada, ainda que poucas espécies com elevada densidade e a maioria com poucos indivíduos $(29,69 \%$ das espécies estão representadas por somente um indivíduo e $68,75 \%$ por seis indivíduos ou menos), apresentando assim um padrão comumente encontrado em florestas tropicais e subtropicais, onde a menor dominância numérica favorece a maior diversidade de espécies no ambiente (Silva et al., 2012). Essa elevada diversidade encontrada na vegetação ciliar estudada deve ser considerada como um atributo desejável em projetos de restauração ativa de matas ciliares na região.

\section{CONCLUSÃO}

Neste trabalho foram fornecidas informações florísticas e fitossociológicas do componente arbóreo-arbustivo de uma mata ciliar em estágio sucessional inicial que podem subsidiar futuros projetos de restauração ecológica em ambientes ciliares na região da área de estudo.

Dentre as espécies prioritárias para uso em plantios de restauração, destacam-se Myrcia selloi, Gymnanthes klotzschiana, Myrcia subcordata, Sebastiania brasiliensis, Myrcia glomerata e Eugenia involucrata que possuem bom desenvolvimento em ambientes ripários e apresentaram abundância e frequência relativamente elevadas neste trabalho e em outros realizados em Floresta Estacional Semidecidual ribeirinha. A elevada riqueza e abundância da família Myrtaceae, bem como a diversidade relativamente elevada e a predominância de espécies zoocóricas e não-pioneiras constituem características marcantes da mata ciliar estudada. Assim, sugere-se que tais características sejam consideradas na modelagem de projetos de restauração ativa de matas ciliares na região. 


\section{AGRADECIMENTOS}

À Coordenação de Aperfeiçoamento de Pessoal de Nível Superior (CAPES) pelo apoio financeiro fornecido para o desenvolvimento deste estudo no âmbito do Programa de Pós-Graduação em Tecnologias Ambientais (PPGTAMB) da Universidade Tecnológica Federal do Paraná (UTFPR). Ao Sr. Rogério Ampessan (Seu Neca) e à comunidade Xaxim, por fornecerem acesso à área de estudo e informações sobre o histórico de uso do solo na área.

\section{BIBLIOGRAFIA}

Accioly, P. (2013). Mapeamento dos remanescentes vegetais arbóreos do estado do Paraná e elaboração de um sistema de informações geográficas para fins de análise ambiental do estado. (Tese de Doutorado), Universidade Federal do Paraná, Curitiba.

Alvares, C. A., Stape, J. L., Sentelhas, P. C., Gonçalves, J. L. M. e Sparovek, G. (2013) Köppen's climate classification map for Brazil. Meteorologische Zeitschrift 22 (6): 711-728.

Angiosperm Phylogeny Group - APG. (2016). An update of the Angiosperm Phylogeny Group classification for the orders and families of flowering plants: APG IV. Botanical Fournal Of The Linnean Society 181 (1): 1-20.

Aquino, C. de e Barbosa, L. M. (2009). Classes sucessionais e síndromes de dispersão de espécie arbóreas e arbustivas existentes em vegetação ciliar remanescente (Conchal, SP), como subsídio para avaliar o potencial do fragmento como fonte de propágulos para enriquecimento de áreas revegetadas no Rio Mogi-Guaçu, SP. Revista Árvore 33 (2): 349-358.

Aronson, J., Durigan, G. e Brancalion, P. H. S. (2011). Conceitos e definições correlatos à ciência e à prática da restauração ecológica. IF Série Registros 44: 1-38.

Barbosa, L. M., Shirasuna, R. T., Lima, F. C. de, Ortiz P. R. T., Barbosa, K. C. e Barbosa T. C. (2017). Lista de espécies indicadas para restauração ecológica para diversas regiões do Estado de São Paulo. São Paulo: Instituto de Botânica.

Bhering, S. B., Santos, H. G. D., Manzatto, C. V., Bognola, I. A., Fasolo, P. J., Carvalho, A. P. D., Potter, R.O. e Curcio, G. R. (2007). Mapa de solos do Estado do Paraná. Rio de Janeiro: Embrapa Solos.

Bianchini, E., Popolo, R. S., Dias, M. C. e Pimenta, J. A. (2003). Diversidade e estrutura de espécies arbóreas em área alagável do município de Londrina, Sul do Brasil. Acta Botanica Brasilica 17 (3): 405-419.

Borghi, W. A., Martins, S. S., Del Quiqui, E. M. e Nanni M. R. (2004). Caracterização e avaliação da mata ciliar à montante da Hidrelétrica de Rosana, na Estação Ecológica do Caiuá, Diamante do Norte, PR. Cadernos da Biodiversidade 4 (2): 9-18.

Brasil. (2012). Lei $\mathrm{n}^{0} 12.651$, de 25 de maio de 2012. Dispõe sobre a proteção da vegetação nativa; altera as Leis nos 6.938, de 31 de agosto de 1981, 9.393, de 19 de dezembro de 1996, e 11.428, de 22 de dezembro de 2006; revoga as Leis nos 4.771, de 15 de setembro de 1965, e 7.754, de 14 de abril de 1989, e a Medida 
Provisória no 2.166-67, de 24 de agosto de 2001; e dá outras providências. Diário Oficial da União.

Brasil. (2014). Portaria $n^{\circ} 443$, de 17 de dezembro de 2014. Lista Nacional Oficial de Espécies da Flora Ameaçadas de Extinção. Diário Oficial da União, Seção 1: $110-121$.

Chaves, R. B., Durigan, G., Brancalion, P. H. S. e Aronson, J. (2015). On the need of legal frameworks for assessing restoration projects success: new perspectives from São Paulo state (Brazil). Restoration Ecology 23 (6): 754-759.

Cielo-Filho, R., Baitello, J. B., Pastore, J. A., Aguiar, O. T. de, Souza, S. C. P. M. de, Toniato, M. T. Z., Lima, C. R. de e Ribeiro, A. P. (2009). Ampliando a densidade de coletas botânicas na região da bacia hidrográfica do Alto Paranapanema: Caracterização florística da Floresta Estadual e da Estação Ecológica de Paranapanema. Biota Neotropica 9 (3): 255-276.

Cielo-Filho, R., Franco, G. A. D. C., Periotto, F., Aguiar, O. T. de, Beitello, J. B., Câmara, C. D., Sousa, C. R. e Jesus, J. M. de (2017a). Tree and shrub flora in the surroundings of the Parque Nacional do Iguaçu, Paraná State , Brazil/ : contribution to ecological restoration. Hoehnea 44 (4): 473-489.

Cielo-Filho, R., Baitello, J. B., Toniato, M. T. Z., Kanashiro, M. M., Mattos, I. F. de A., Aguiar, O. T. de, Souza, S. C. P. M. de, Zimback, L. e Franco, G. A. D. C. (2017b). A vegetação da Estação Ecológica de Paranapanema: subsídios para o plano de manejo. IF Série Registros 55: 75-127.

Delucchi, G. e Keller, H. A. (2010). La naturalización del «níspero», Eriobotrya japonica (Rosaceae, Maloideae), en la Argentina. Bonplandia 19 (1): 71-77.

Dias, M. C., Vieira, A. O. S. e Paiva, M. R. C. (2002). Florística e fitossociologia das espécies arbóreas das florestas da bacia do rio Tibagi. In: Medri, M. E., Bianchini, E., Shibatta, O. A. e Pimenta, J. A. (Eds.). A Bacia do Rio Tibagi (pp. 109-124). Londrina.

Di Bitetti, M. S., Placci, G. e Dietz, L. A. (2003). Uma visão de Biodiversidade para a Ecorregião Florestas do Alto Paraná - Bioma Mata Atlântica: planejando a paisagem de conservação da biodiversidade e estabelecendo prioridades para ações de conservação. Washington, D.C.: World Wildlife Fund.

Estevan, D. A., Vieira, A. O. S. e Gorenstein, M. R. (2016). Estrutura e Relações Florísticas de um Fragmento de Floresta Estacional Semidecidual, Londrina, Paraná, Brasil. Ciência Florestal 26 (3): 713-725.

Flora do Brasil 2020. (2021). Jardim Botânico do Rio de Janeiro. Recuperado de http://floradobrasil.jbrj.gov.br/

Forzza, R. C., Baumgratz, J. F. A., Bicudo, C. E. M., Canhos, D. A. L., Carvalho Jr., A. A., Coelho, M. A. N., Costa, A. F., Costa, D. P., Hopkins, M. G., Leitman, P. M., Lohmann, L. G., Lughadha, E. N., Maia, L. C., Martinelli, G., Menezes, M., Morim, M. P., Peixoto, A. L., Pirani, J. R., Prado, J., Queiroz, L. P., Souza, S., Souza, V. C., Stehmann, J. R., Sylvestre, L. S., Walter, B. M. T. e Zappi, D. C. (2012). New brazilian floristic list highlights conservation challenges. Bioscience 62 (1): 39-45.

Freitas, W. K. e Magalhães L. M. S. (2012). Métodos e parâmetros para estudo da vegetação com ênfase no estrato arbóreo. Floresta e Ambiente 19 (4): 520-540. 
Gris, D. e Temponi, L. G. (2017). Similaridade florística entre trechos de Floresta Estacional Semidecidual do corredor de biodiversidade Santa Maria - PR. Ciência Florestal 27 (3): 1069-1081.

Hornes, K. L. e Rodrigues, E. D. A. (2018). Fitogeografia da Bacia Hidrográfica do Paraná 3. In: Rocha, A. S. D. e Bade, M. R. (Orgs). Geografia da Bacia Hidrográfica do Paraná 3: fragilidades e potencialidades socioambientais (pp. 99-119). Jundiaí: In House.

Instituto Brasileiro de Geografia e Estatística - IBGE (2012). Manual técnico da vegetação brasileira: sistema fitogeográfico, inventário das formações florestais e campestres, técnicas e manejo de coleções botânicas, procedimento para mapeamento. Rio de Janeiro: IBGE - Coordenação de Recursos Naturais e Estudos Ambientais.

Instituto Hórus de Desenvolvimento e Conservação Ambiental (2021). Base de dados de espécies exóticas invasoras do Brasil. Florianópolis - SC. Recuperado de http://bd.institutohorus.org.br/www/

Kieras, W. S., Accioly, Y. e Machado, S. A. (2018). Análise estrutural e dinâmica de Gymnanthes klotzschiana (Müll. Arg.) em um fragmento de Floresta Ombrófila Mista. Scientia Agraria Paranaensis 17 (1): 71-79.

Kinoshita, L. S., Torres, R. B., Forni-Martins, E. R., Spinelli, T., Ahn, Y. J. e Constâncio, S. S. (2006). Composição florística e síndromes de polinização e de dispersão da mata do Sítio São Francisco, Campinas, SP, Brasil. Acta Botanica Brasilica 20 (2): 313-327.

Lazzarin, L. C., Silva, A. C., Higuchi, P., Souza, K., Perin, J. E. e Cruz, A. P. (2015) Invasão biológica por Hovenia dulcis Thunb. em fragmentos florestais na região do alto Uruguai, Brasil. Revista Árvore 39: 1007-1017.

Liebsch, D., Marques, M. C. M. e Goldenberg, R. (2008). How long does the Atlantic Rain Forest take to recover after a disturbance? Changes in species composition and ecological features during secondary succession. Biological Conservation 141 (6): 1717-1725.

Lisboa, T. F. B., Cielo-Filho, R. e Câmara, C. D. (2021). Applicability of monitoring protocols developed for active restoration projects in the evaluation of passive restoration of a subtropical riparian forest in Brazil. Tropical Ecology 62 (1): $17-26$.

Ludwig, J. A. e Reynolds, J. F. (1988). Statistical ecology: A primer on methods and computing. John Wiley \& Sons: New York.

Machado, E. L. M., Oliveira-Filho, A. T., Berg, E. V. D., Carvalho, W. A. C., Souza, J. S., Marques, J. J. G. S. M. e Calegário, N. (2008). Efeitos do substrato, bordas e proximidade espacial na estrutura da comunidade arbórea de um fragmento florestal em Lavras, MG. Revista Brasileira de Botânica 31 (2): 287-302.

Maciel, M. de N. M., Watzlawick, L. F., Schoeninger, E. R. e Yamaji, F. M. (2003). Classificação ecológica das espécies arbóreas. Revista Acadêmica: Ciências Agrárias e Ambientais 1 (2): 69-78.

Meira Junior, M. S., Pereira, I. M., Machado, E. L. M., Mota, S. L. L. e Otoni, T. J. O. (2015). Espécies potenciais para recuperação de áreas de Floresta Estacional 
Semidecidual com exploração de minério de ferro na Serra do Espinhaço. Bioscience Fournal 31 (1): 283-295.

Mello, K., Petri, L., Leite, E. C. e Toppa, R. H. (2014). Cenários ambientais para o ordenamento territorial de áreas de preservação permanente no município de Sorocaba, SP. Revista Árvore 38 (2): 309-317.

Melo, A. C. G. e Durigan, G. (2007). Evolução estrutural de reflorestamentos de restauração de matas ciliares no Médio Vale do Paranapanema. Scientia Forestalis 73: 101-111.

Mikich, S. B. e Silva, S. M. (2001). Composição florística e fenologia das espécies zoocóricas de remanescentes de floresta estacional semidecidual no centro-oeste do Paraná, Brasil. Acta Botanica Brasilica 15 (1): 89-113.

Moro, M. F. e Martins, F. R. (2011). Métodos de levantamento do componente arbóreo-arbustivo. In: Felfili, J. M., Eisenlohr, P. V., Melo, M. M. da R. F. de, Andrade, L. A. de e Neto, J. A. A. M. (Eds.). Fitossociologia no Brasil: Métodos e Estudos de Caso (pp. 174-212). Viçosa: Editora UFV.

Moro, M. F., Souza, V. C., Oliveira-Filho, A, T. de, Queiroz, L. P. de, Fraga, C. N. de; Rodal, M. J. N., Araújo, F. S. de, Martins, F. R. (2012). Alienígenas na sala: o que fazer com espécies exóticas em trabalhos de taxonomia, florística e fitossociologia? Acta Botanica Brasilica 26 (4): 991-999.

Muchailh, M. C., Roderjan, C. V., Campos, J. B., Machado, A. L. T. e Curcio, G. R. (2010). Metodologia de planejamento de paisagens fragmentadas visando a formação de corredores ecológicos. Revista Floresta 40 (1): 147-162.

Myers, N., Mittermeier, R. A., Mittermeier, C. G., Fonseca, G. A. B. e Kent, J. (2000). Biodiversity hotspots for conservation priorities. Nature 403: 853-858.

Nitsche, P. R., Caramori, P. H., Ricce, W. S. e Pinto, L. F. D. (2019). Atlas climático do Estado do Paraná. Londrina: Instituto Agronômico do Paraná.

Oliva, E. V., Reissmann, C. B., Marques, R., Bianchin, J. E., Dalmaso, C. A. e Winagraski, E. (2018). Florística e estrutura de duas comunidades arbóreas secundárias com diferentes graus de distúrbio em processo de recuperação. Ciência Florestal 28 (3): 1088-1103.

Paraná. Instituto Ambiental do Paraná - IAP. (2015). Portaria IAP n 59, de 15 de abril de 2015. Reconhece como espécies exóticas invasoras no estado do Paraná as espécies relacionadas nos Anexos 1 (Plantas), 2 (Vertebrados) e 3 (Invertebrados) da presente Portaria. Diário Oficial do Estado.

Ramos, V. S., Durigan, G., Franco, G. A. D. C., Siqueira, M. F. e Rodrigues, R. R. (2015). Árvores da Floresta Estacional Semidecidual: guia de identificação de espécies. EDUSP.

Rodrigues, R. R. (2000). Florestas Ciliares: Uma discussão nomenclatural das formações ciliares. In: Rodrigues, R. R., Leitão-Filho, H.F. (Orgs.). Matas Ciliares: conservação e recuperação (pp. 91-99). EDUSP/FAPESP.

Scariot, E. C. e Reis, A. (2010). Riqueza e estrutura florística de corredores ciliares em regeneração natural no planalto norte catarinense, Sul do Brasil. Perspectiva 34 (125): 53-65. 
Seganfredo, D., Cunha, B., Magalhães, V. L., Cielo-Filho, R. e Câmara, C. D. (2019). Landscape Ecology in a Watershed of the Ocoy River, Western Parana State, Brazil. Floresta e Ambiente 26 (1): 1-10.

Silva, F. das C. e, Fonseca, E. de P., Soares-Silva, L. H., Muller, C. e Bianchini, E. (1995). Composição florística e fitossociologia do componente arbóreo das florestas ciliares da Bacia do Rio Tibagi. 3. Fazenda Bom Sucesso, Município de Sapopema, PR. Acta Botanica Brasilica 9 (2): 289-302.

Silva, A. C. da, Higuchi, P., Aguiar, M. D. de, Negrini, M., Neto, J. F. e Hess, A. F. (2012). Relações florísticas e fitossociologia de uma Floresta Ombrófila Mista Montana Secundária em Lages, Santa Catarina. Ciência Florestal 22 (1):193206.

Society For Ecological Restoration - SER (2004). International Science and Policy Working Group. The SER primer on ecological restoration (Version 2).

Souto, M. A. G. e Boeger, M. R. T. (2011). Estrutura e composição do estrato de regeneração e vegetação associada de diferentes estádios sucessionais no Leste do Paraná. Ciência Florestal 21 (3): 393-406.

Souza, R. F., Machado, S. A., Galvão, F. e Filho, A. F. (2017). Fitossociologia da vegetação arbórea do Parque Nacional do Iguaçu. Ciência Florestal 27 (3): 853-869.

Stampella, P. C., Delucchi, G. e Pochettino, M. L. (2013) Naturalización e identidad del "limón mandarina", Citrus $\times$ taitensis (Rutaceae, Aurantioideae) en la Argentina. Boletín de la Sociedad Argentina de Botánica 48: 161-169.

Tossulino, M. G. P., Siqueira, J. D. P., Morato, S. A. A., Sayama, C., Ulandowski, L. K. M. A., Cavilha, M. R. e Schitza, E. G. (Orgs.) (2007). Resumo executivo da avaliação ecológica rápida do Corredor Iguaçu-Paraná. Curitiba: IAP.

Tundisi, J. G. e Tundisi, T. M. (2010). Impactos potenciais das alterações do Código Florestal nos recursos hídricos. Biota Neotropica 10 (4): 67-75.

Van der Pijl, L. (1982). Principles of dispersal in higher plants. Berlin: SpringerVerlag.

Viapiana, J., Cielo-Filho, R. e Câmara, C. D. (2019). Evaluation and characterization of passive restoration of an Atlantic forest tree community with focus on the understory. Tropical Ecology 60 (1): 140-149.

Whitmore, T. C. (1989). Canopy gaps and the two major groups of forest trees. Eco$\log 70$ (3): 536-538.

Yamamoto, L. F, Kinoshita, L. S e Martins, F. R. (2005). Florística dos componentes arbóreo e arbustivo de um trecho da Floresta Estacional Semidecídua Montana, município de Pedreira, estado de São Paulo. Revista Brasileira de Botânica 28 (1): 191-202. 